\title{
ERRORS IN SIMULTANEOUS LINEAR EQUATIONS ${ }^{1}$
}

BY RAYMOND REDHEFFER (Research Lab. of Electronics, M. I. T.)

1. The problem. After computing the unknowns in a linear system $\sum_{1}^{n} a_{i j} x_{i}=b_{i}$ with non-vanishing determinant, one may check the work by substitution into the original equations. If the $j$-th equation is satisfied within an error $e_{i}$, it is natural to inquire into what can be said about the corresponding error in the unknowns. This question is the subject of the present note. Analytically we have

$$
\sum a_{i j}\left(x_{i}+E_{i}\right)=b_{i}+e_{i},
$$

where $x_{i}$ are the exact values of the unknowns, and where the errors $E_{i}$ in the unknowns are to be estimated in terms of the substitution errors $e_{i}$. This problem was suggested by Prof. P. Franklin.

2. A solution. Regarding $\left\{E_{i}\right\}$ and $\left\{e_{i}\right\}$ as vectors, let us estimate the maximum length of one in terms of the length of the other; in other words let us find $\max \left(\sum E_{i}^{2}\right)^{1 / 2}$ subject to $\left(\sum e_{i}^{2}\right)^{1 / 2}=e$. This is equivalent to finding the minimum of $\sum e_{i}^{2}$ subject to a constant value of $\sum E_{i}^{2}$, and such a problem lends itself to standard methods. Using the fact that the $x_{i}$ satisfy the equations exactly, we have

$$
\sum a_{i j} E_{i}=e_{j} .
$$

We are thus required to minimize

$$
\sum_{i}\left(\sum_{i} a_{i j} E_{i}\right)^{2}
$$

subject to the condition that $\sum E_{i}^{2}=$ constant. By collecting terms in (3) we find that the matrix of the quadratic form is $A A^{\prime}$, where $A=\left(a_{i j}\right)$ is the matrix of the original system and $A^{\prime}$ is its transpose. Hence, if $e$ is the length of the known error vector, $e=$ $\left(\sum e_{i}^{2}\right)^{1 / 2}$, and $E$ is the length of the unknown error vector, $E=\left(\sum E_{i}^{2}\right)^{1 / 2}$, then we have ${ }^{1}$

$$
E \leq e /\left(\lambda_{m}\right)^{1 / 2}
$$

where $\lambda_{m}$ is the minimum characteristic value of the matrix $A A^{\prime}$. When the matrix $A$ is symmetrical, in particular, we have

$$
E \leq e /\left|\lambda_{m}^{\prime}\right|,
$$

where $\lambda_{m}^{\prime}$ is (in absolute value) the minimum characteristic value of $A$. These results are the best possible, in that equality is always attained, for given $e$, with some set of values $e_{i}$.

3. Approximation. If $\lambda_{\imath}$ are the characteristic values of $A A^{\prime}$ we know that

$$
\sum \lambda_{i}=\sum \sum a_{i j}^{2}=T,
$$

the trace of $A A^{\prime}$. Also

$$
\Pi \lambda_{i}=|A|^{2}
$$

${ }^{1}$ Received Nov. 21, 1947. This work has been supported in part by the Signal Corps, the Air Materiel Command, and the Office of Naval Research.

${ }^{2}$ R. Courant and D. Hilbert, Methoden der Mathematischen Physik, vol. 1, J. Springer, Berlin, 1931, p. 21. 
if $|A|$ stands for the determinant of $A$. By (3) it is clear that $A A^{\prime}$ is positive definite, so that $\lambda_{i}>0$, and hence we may use (6) to find

$$
\sum_{i \neq m} \lambda_{i}<T
$$

while in any case we have, by (7),

$$
\lambda_{m} \prod_{i \neq m} \lambda_{i}=|A|^{2} .
$$

If the sum of $n$ positive quantities is constant the product is maximum when they are all equal. Hence we find, by (8) and (9),

$$
\lambda_{m}>\frac{|A|^{2}}{T^{n-1}}(n-1)^{n-1}
$$

with the final result

$$
E<\frac{e}{\|A\|}\left(\frac{T}{n-1}\right)^{(n-1) / 2}
$$

which is not difficult to compute numerically.

From the derivation it is clear that this upper bound is not far from optimum, whenever $\lambda_{m}$ is small compared to the sum of the $\lambda_{i}$, and in addition the other $\lambda_{i}$ do not vary over too wide a range of values. In the case of any normal orthogonal transformation, for example, we have

$$
E<\frac{e}{1}\left(\frac{n}{n-1}\right)^{(n-1) / 2} \rightarrow e(2.718 \cdots)^{1 / 2} \cong 1.65 e
$$

by (11), whereas the optimum inequality is $E \leq e$.

4. Numerical examples. Suppose that approximate values of the unknowns are substituted in a system with the matrix

$$
A=\left(\begin{array}{rrr}
3 & -2 & -2 \\
-2 & 5 & 1 \\
-2 & 1 & 4
\end{array}\right)
$$

and are found to satisfy the equations with errors of $0.1,0.1$ and 0.2 respectively. To find the maximum possible error in any one unknown we proceed as follows. The determinant of the system $A$ is 29 , and for $T$ we take the sum of the squares of the entries in (12), which gives $T=68$. Substituting in (11) with $n=3$, we find

$$
E<1.17 e
$$

In the present case $e=(.01+.01+.04)^{1 / 2}=.22$ so that $E$, and hence the maximum error in any one unknown, cannot exceed 0.26 .

The characteristic values were found by J. G. Linvil to be $1.13,7.39$, and 3.49. Using $\lambda_{m}=1.13$ in (5) we obtain the optimum inequality

$$
E<0.89 e
$$

which is only a slight improvement on (13). 